\title{
Acuicultura Integrada Multi-Trófica (IMTA) y sus desafíos para el secuestro de carbono y biomitigación de procesos de eutrofización costera
}

\author{
Buschmann A. H. *, Hernández-González M. C. \\ Centro i mar y CeBiB, Universidad de Los Lagos, Camino a Chinquihue km 6, Puerto Montt, Chile \\ *E-mail: abuschma@ulagos.cl
}

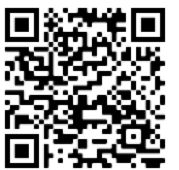

Cite this paper/Como citar este artículo: Buschmann A. H., Hernández-González M. C. (2021). Acuicultura Integrada Multi-Trófica (IMTA) y sus desafíos para el secuestro de carbono y biomitigación de procesos de eutrofización costera. Revista Bio Ciencias 8: (Suppl) Memorias del 3er Coloquio de Nutrigenómica y Biotecnología Acuícola 2020 (CONYBA) e1182. http://doi.org/10.15741/revbio.08Suppl.e1182

\begin{abstract}
Resumen
Este trabajo revisa el desarrollo del concepto de Acuicultura Integrada Multi-Trófica (IMTA) y sus beneficios ambientales y productivos. Analiza los avances y restricciones que existen actualmente y plantea la necesidad de establecer sistemas demostrativos a escala comercial, incorporando sus externalidades ambientales. Se plantean los beneficios de su adopción en acuicultura para un desarrollo más sustentable de ésta, tanto en los cuerpos de agua dulce como marina.
\end{abstract}

\section{PALABRAS CLAVE}

$\begin{array}{lcc}\text { Acuicultura } & \text { integrada } & \text { Multi-Troficas, } \\ \text { Biorremediación } & \text { Ambiental, } & \text { Eutrofización, }\end{array}$ Secuestro de Carbono

\section{Introducción}

Conceptualmente, la Acuicultura Integrada MultiTrófica o IMTA, consiste en cultivar especies de distinto nivel trófico en proximidad, de tal modo que tengan funciones ecosistémicas complementarias. Esto permite que los desechos orgánicos e inorgánicos de especies consumidoras (e.g. peces carnívoros) puedan ser asimilados por especies extractivas (e.g. moluscos filtradores, especies detritívoras y algas). Al recapturar y convertir los desechos y energía producida por especies consumidoras, se produce un efecto positivo de biomitigación de los impactos ambientales que supone la introducción de estos compuestos en ecosistemas dulceacuícolas y marinos. Adicionalmente es posible obtener beneficios económicos complementarios con la producción de diferentes especies y a futuro permite pensar en bonos de carbono o bonos de nitrógeno que pudieran aumentar la rentabilidad de estas prácticas de acuicultura (Chopin \& Tacon, 2020).
En este trabajo se resumen etapas del desarrollo de la Acuicultura Integrada Multi-Trófica, hitos y desafíos tanto alcanzados como no alcanzados (Tabla 1). El concepto de IMTA data desde épocas anteriores a 2000 A.C. donde, en China, ya se menciona el cultivo de peces con plantas acuáticas (Chopin, 2013). También se describen sistemas integrados de agricultura y acuicultura de tilapia en Egipto, hacia el año 1500 A.C. En Occidente, sólo en la época del renacimiento en Francia, se instaló la producción de carpas en un sistema autosustentable donde la presencia de otras especies permitían la mantención de los peces. Sin embargo, en la edad contemporánea, es sólo hacia 1975 cuando el Dr. John Ryther, comienza estudios con base científica y técnica para permitir la recirculación de desechos en sistemas marinos de policultivos, primero en Woods Hole Oceanographic Institution, en Massachusetts y luego trasladándose al Harbor Branch Oceanographic Institute de Florida, en Estados Unidos. A partir de estos trabajos, el concepto de IMTA comienza a incorporarse en otros estudios realizados en Estados Unidos, Israel, Chile, Suecia y Canadá. Entre los años 1999, y 2014 se publican 6 revisiones que impactan fuertemente en el desarrollo de posteriores trabajos sobre IMTA en el mundo (Troell et al., 1999; 2003; Buschmann et al., 2001; Chopin et al., 2001; Fei, 2004; Neori et al., 2004).

\section{Desarrollo del Concepto de Acuicultura} Integrada Mutitrófica

Actualmente la acuicultura constituye la principal fuente de productos del mar para alimentación humana, siendo, de acuerdo a las estadísticas de la FAO, superior al aporte que tiene la pesca (Chopin \& Tacon, 2020). Considerando diversos y complejos efectos ambientales que la acuicultura puede tener sobre sistemas dulceacuícolas y 
marinos, el desarrollo de IMTA permite, al menos, disminuir los efectos de los aportes de nutrientes orgánicos e inorgánicos al medio ambiente (Chopin et al., 2008). Estudios de balance de masa indican que cuando se cosechan especies consumidoras como peces cultivados en sistemas de balsa jaula, del $100 \%$ del nitrógeno y fósforo introducido en el alimento, sólo un $25 \%$ se recupera al cosechar los peces (e.g. Buschmann et al., 2009). Del 75\% que permanece en el medio ambiente, el $65 \%$ del fósforo sedimenta bajo los sistemas de cultivo. En tanto que para el caso del nitrógeno, a los sedimentos sólo se incorpora el $15 \%$. Los compuestos nitrogenados mayoritariamente (60\%), se integran a la columna de agua liberados en los procesos de excreción de los peces cultivados. Este nitrógeno inorgánico disuelto en la zona fótica de la columna de agua, queda disponible para el crecimiento de micro y macroalgas. Un estudio experimental realizado hacia mediados de 1990 demostró que el alga Agarophyton chilense (previamente identificada como Gracilaria chilensis), al ser cultivada en las cercanías de balsas jaulas de cultivos de salmón, presentaba tasas de crecimiento significativamente mayores, así como mayor acumulación de nitrógeno y fósforo en sus tejidos, en comparación a cuando ésta era cultivada a unos 500 metros de distancia del cultivo de peces (Troell et al., 1997). En el mencionado trabajo, la escala de producción de salmones no superaba las 400 toneladas anuales. Al repetir este tipo de estudios cultivando también el alga roja Agarophyton chilense, fue posible determinar que cuando el centro de cultivo de peces tiene una producción de 1500 toneladas anuales, el alga puede mantener un incremento significativo de su crecimiento y de acumulación de nitrógeno en los tejidos hasta una distancia cercana a 1 kilómetro (Abreu et al., 2009). Esta evidencia indica que al aumentar la escala de producción, los efectos ambientales de la introducción de $\mathrm{N}$ inorgánico puede alcanzar una amplia zona alrededor de un centro de cultivo. De aquí la relevancia del cultivo de algas al mitigar la introducción de $\mathrm{N}$ en los ecosistemas costeros, que podría desencadenar procesos de eutrofización, dado que este es el nutriente que generalmente limita la productividad primaria en sistemas costeros.

Numerosos estudios han demostrado la capacidad de organismos filtradores y de algas marinas para capturar material orgánico particulado y nutrientes inorgánicos disueltos. Las aproximaciones experimentales, en general, han permitido determinar como el flujo de materia y energía, proveniente de especies consumidoras, puede ser reutilizado por los organismos extractivos. Un análisis bibliográfico utilizando la Web of Knowledge

(Wok,https://apps.webofknowledge.com/WOS Ge neralSearch ), permite puntualizar que entre los años 1975 y octubre de 2020, de las 4430 publicaciones científicas sobre IMTA, solo 126 contienen información sobre captura de nitrógeno. Sin embargo, dada la escala relativamente pequeña en las que los organismos extractivos son estudiados en sistemas de IMTA, en general estos trabajos no permiten determinar los efectos de estos sobre las especies consumidoras ni de los beneficios ambientales que generan. Para demostrar estos efectos, se necesitan instalaciones a escala pre-comercial y/o comercial, que logren demostrar que el uso de tecnología IMTA, para la producción acuícola, puede tener efectos significativos sobre los ecosistemas dulceacuícolas y marinos. A modo de ejemplo, se ha estimado que se requieren más de 100 hectáreas de cultivo de algas para biorremediar en un porcentaje significativo, la producción de 1000 toneladas anuales de peces. Es necesario, por tanto, obtener recursos que permitan realizar ensayos a nivel comercial y demostrar los beneficios ambientales y productivos de una acuicultura recirculante, en la que se extraen gran cantidad de desechos de los ecosistemas utilizados para realizar acuicultura.

A pesar de las restricciones ya indicadas, hay que incorporar y considerar la información que en los últimos años emerge de experiencias en regiones y países donde la producción de organismos extractivos como bivalvos filtradores y algas es muy significativa. Estudios recientes de Xiao et al. (2017) señalan que en China, el cultivo a gran escala (miles hectáreas) de Nori (Pyropia), ha permitido reducir las cargas de nitrógeno inorgánico producido por variadas actividades humanas que descargan sus desechos en zonas costeras. Por otra parte, Yang et al. (2015) evidencia cómo el cultivo de Gracilaria, también a gran escala, permite reducir las cargas de nutrientes disueltos, disminuyendo la concentración de fitoplancton, aumentando la transparencia del agua y bajando la frecuencia e intensidad de mareas rojas. Esta evidencia merece ser analizada con mayor detalle en países occidentales donde la acuicultura basada en organismos consumidores (camarones y peces carnívoros) está teniendo mayor desarrollo.

En occidente si bien existen considerables avances en producción de bivalvos filtradores y algas utilizando efluentes de peces, quizás el ejemplo comercial más relevante lo encontramos en África del Sur. Allí se han desarrollado sistemas de acuicultura a escala comercial, de abalones y el alga verde Ulva sp. Basado en un sistema de cultivo piloto de Ulva lactuca que utilizaba el $25 \%$ del agua proveniente de sistemas de cultivo del abalón Haliotis midae, fue posible tener una fuente sustentable de alimento para la producción de abalón, con beneficios adicionales como la oxigenación del agua y remoción de nitrógeno permitiendo recircular el agua y siendo, además, una fuente real de generación de empleo en lugares apartados del país (Troell et al., 2006). Este es considerado el primer sistema planificado de IMTA a gran escala. 
En los últimos años se ha argumentado que, además de disminuir las cargas de nitrógeno provenientes de actividades de acuicultura de organismos consumidores, transforman los ecosistemas dulceacuícolas y marinos desde estados oligotróficos hacia estados mesotróficos e incluso eutróficos. También se ha señalado que la presencia de bivalvos filtradores y algas, al permitir secuestro de carbono, puede considerarse una herramienta para disminuir procesos de acidificación oceánica (Chopin \& Tacon, 2020). Un análisis bibliográfico en la WoK permite establecer que desde 1975 a octubre de 2020 se han producido 4430 publicaciones científicas sobre IMTA, sin embargo, solo en 39 de éstas relacionan la Acuicultura Integrada Multi-Trófica con la acidificación oceánica. Actualmente se requiere información científica validada sobre cómo las emisiones antropogénicas de dióxido de carbono $\left(\mathrm{CO}_{2}\right)$ pueden ser secuestradas en sistemas de IMTA, evitando o reduciendo la acidificación de los sistemas costeros.

\section{Conclusiones}

El concepto de IMTA tiene un origen que remonta hacia épocas A.C., pero sólo hace unas tres décadas, que se comienzan a establecer sus bases científicas, permitiendo así su desarrollo. En principio es un concepto que aúna el cultivo de especies consumidoras y extractivas para producir beneficios ambientales económicos y sociales. Este trabajo plantea que el desarrollo de IMTA tiene todavía el desafío de demostrar que puede generar beneficios ambientales a grandes escalas espaciales y temporales (cientos a miles de hectáreas). La evidencia más reciente indica que, al menos en sistemas de cultivo abierto, el cultivo de algas está demostrando beneficios ambientales y es esperable que el desarrollo de sistemas de recirculación como el ejemplo de África del Sur pueda ser replicado en otras regiones del planeta. Un reto fundamental es lograr determinar los impactos de sistemas de IMTA con respecto a la posible mitigación de cambio climático por medio del secuestro de carbono.

\section{Agradecimientos}

En más de treinta años de trabajo son muchos los colegas y estudiantes que han aportado a esta línea de trabajo. A todos ellos nuestros agradecimientos. Fondos para realizar estos trabajos han sido entregados por FONDECYT, FONDEF, así como también por los aportes del Programa Basal que soporta al Centro de Biotecnología y Bioingeniería, (CeBiB, FB-001)) de la Agencia Nacional de Ciencias y Desarrollo de Chile (ANID).

Tabla 1. Desarrollo del concepto de Acuicultura Integrada Multi-Trófica (IMTA). Tabla basada en Chopin 2013 y literatura citada en Neori et al. 2004.

\begin{tabular}{|c|c|}
\hline \multirow{2}{*}{\multicolumn{2}{|c|}{ Pasado "Antiguo" (Antes de Cristo) }} \\
\hline & \\
\hline 2200-2100 A.C. & $\begin{array}{l}\text { You Ho Bin detalla en sus manuscritos la integración de peces con } \\
\text { la producción de plantas acuáticas en China. }\end{array}$ \\
\hline 1970-1780 A.C & Registros del cultivo de peces en arrozales en China. \\
\hline 1550-1070 A.C. & $\begin{array}{l}\text { Registros del cultivo de tilapia en Egipto en sistemas de drenaje } \\
\text { integrando agricultura y acuicultura. }\end{array}$ \\
\hline 1330-1100 A.C. & $\begin{array}{l}\text { Se registra en documentos el desarrollo de los sistemas de } \\
\text { policutivo asociado a sistemas agrícolas en China. }\end{array}$ \\
\hline \multicolumn{2}{|l|}{ Pasado (Despúes de Cristo) } \\
\hline $889-904$ & $\begin{array}{l}\text { Liu Xun publica en China "The Curious of Lingbiao Region" donde } \\
\text { se describe la simbiosis entre carpas y arrozales, así como los } \\
\text { beneficios del cultivo de peces con frutales. }\end{array}$ \\
\hline 1500 & Registro de policultivo en piletas en Java del Este. \\
\hline 1600 & $\begin{array}{l}\text { Por solicitud del rey de Francia, Enrique IV, se construye el Château } \\
\text { de Fointainebleau, un sistema de estanques para carpas } \\
\text { ambientalmente autosustentable. }\end{array}$ \\
\hline 1639 & $\begin{array}{l}\text { Xu Guangpi publica "The Complete Book of Agriculture" donde } \\
\text { describe la rotación de sistemas de irrigación de peces y plantas } \\
\text { acuáticas y la integración de peces con ganado. }\end{array}$ \\
\hline \multicolumn{2}{|l|}{$\begin{array}{l}\text { Primeros conceptos de Acuicultura } \\
\text { Integrada }\end{array}$} \\
\hline $\begin{array}{l}\text { 1975: John Rhyter y colaboradores } \\
\text { (US) }\end{array}$ & $\begin{array}{l}\text { Desarrollo de sistemas integrados con reciclamiento de peces y } \\
\text { algas (ver Rhyter et al., 1975). }\end{array}$ \\
\hline $\begin{array}{l}\text { 1977: Langton y colaboradores (Islas } \\
\text { Virgenes) }\end{array}$ & $\begin{array}{l}\text { Muestran como los aportes nitrogenados de moluscos bivalvos } \\
\text { permiten ser recuperados por un alga roja (Langton et al., 1977). }\end{array}$ \\
\hline $\begin{array}{l}\text { 1979: Marylin Harlin y colaboradores } \\
\text { (US) }\end{array}$ & $\begin{array}{l}\text { Desarrollo de sistema cerrado de algas y peces (ver Harlin et al., } \\
\text { 1979). }\end{array}$ \\
\hline
\end{tabular}




\begin{tabular}{|c|c|}
\hline 1987: M.E. McDonald (US) & $\begin{array}{l}\text { Desarrollo de un sistema de peces y algas para remover nutrientes } \\
\text { (e.g. McDonald, 1987). }\end{array}$ \\
\hline 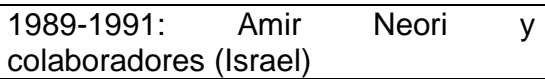 & $\begin{array}{l}\text { Desarrollo del cultivo de algas como biofiltros en cultivos intensivos } \\
\text { (ver Neori et al. 1989, 1991). }\end{array}$ \\
\hline $\begin{array}{l}\text { 1990-1993: Muki Shpigel y } \\
\text { colaboradores }\end{array}$ & $\begin{array}{l}\text { Desarrollo de trabajos en ostras asociados a peces (ver Shpigel et } \\
\text { al., 1990, 1993). }\end{array}$ \\
\hline 1993-1997: Petrell y colaboradores & $\begin{array}{l}\text { Desarrollo de primeras experiencias de uso de algas para mitigar } \\
\text { descargas de compuestos nitrogenados en sistemas abiertos (e.g. } \\
\text { Troell et al., 1997) y de modelos matemáticos integrando flujo de } \\
\text { nutrientes del cultivo de peces y algas (Petrell et al., 1993, Petrell \& } \\
\text { Alie 1996). }\end{array}$ \\
\hline $\begin{array}{l}\text { 1994-1996: Alejandro Buschmann y } \\
\text { colaboradores (Chile) }\end{array}$ & $\begin{array}{l}\text { Cultivo de peces, moluscos filtradores y algas en sistemas de } \\
\text { estanques intensivos de flujo abierto (ver Buschmann et al., 1994; } \\
\text { 1996) }\end{array}$ \\
\hline $\begin{array}{l}\text { 1999-2004: Desarrollo de trabajo } \\
\text { cooperativo entre investigadores de } \\
\text { Canadá, Israel, Chile, Suecia y USA }\end{array}$ & $\begin{array}{l}\text { Integración y consolidación del concepto de que especies } \\
\text { consumidoras y extractivas podian reutilizar desechos, mitigando } \\
\text { sus impactos ambientales y permitiendo una producción } \\
\text { diversificada de la acuicultura (ver trabajos de Troell et al., 1999; } \\
\text { 2003; Buschmann et al., 2001; Chopin et al., 2001; Fei et al., } 2004 \\
\text { y Neori et al., 2004) }\end{array}$ \\
\hline \multicolumn{2}{|l|}{$\begin{array}{l}\text { Desarrollo presente del concepto y } \\
\text { ejemplos de uso y efectos } \\
\text { ambientales de IMTA }\end{array}$} \\
\hline 2004 & $\begin{array}{l}\text { Desarrollo del concepto de Acuicultura Integrada Multi-Trófica por } \\
\text { Thierry Chopin and Jack Taylor y se establece que este concepto } \\
\text { va más allá del co-cultivo de diferentes especies (policultivo) en un } \\
\text { mismo reservorio de agua. }\end{array}$ \\
\hline Bolton & $\begin{array}{l}\text { Se establece a escala comercial un sistema de cultivo de abalones } \\
\text { donde las algas extraen nutrientes disueltos, reciclando los } \\
\text { efluentes (disminución cargas de amonio y } \mathrm{CO}_{2} \text {, aporte de } \mathrm{O}_{2} \text { ) lo } \\
\text { cual permite disminuir el bombeo de agua. }\end{array}$ \\
\hline $2015-2017$ & $\begin{array}{l}\text { Se demuestra a escala comercial (cientos a miles de hectáreas) los } \\
\text { beneficios de la Acuicultura Integrada Multi-Trófica (e.g. Yang et al., } \\
2015 \text {, Xiao et al., 2017) }\end{array}$ \\
\hline
\end{tabular}

\section{Referencias}

Abreu, M. H., Varela, D. A., Henríquez, L., Villaroel, A., Yarish C., Sousa-Pinto, I. and Buschmann, A. H. (2009). Traditional vs. Integrated Multi-Trophic Aquaculture of Gracilaria chilensis (C.J. Bird, J. McLachlan \& E.C. Oliveira: productivity and physiological performance. Aquaculture, 293 (3-4): 211-230. https://doi.org/10.1016/j.aquaculture.2009.03.043

Buschmann, A. H., Mora, O., Gómez, P., Böttger, M., Buitano, S., Retamales, C. A., Vergara P. A. and Gutiérrez, A. (1994). Gracilaria chilensis outdoor tank cultivation in Chile: use of land-based salmon culture effluents. Aquacultural Engineering, 13 (4): 283-300. https://doi.org/10.1016/0144-8609(94)90016-7

Buschmann, A. H., Troell, M., Kautsky, N. and Kautsky, L. (1996). Integrated tank cultivation of salmonids and Gracilaria chilensis (Rhodophyta). Hydrobiologia, 326: 75-82. https://doi.org/10.1007/BF00047789

Buschmann, A. H., Troell, M. and Kautsky, N. (2001). Integrated algal farming: a review. Cahiers de Biologie Marine, 42: 83-90.

Buschmann, A. H., Cabello, F., Young, K., Carvajal, J., Varela, D. A. and Henríquez, L.(2009). Salmon aquaculture and coastal ecosystem health in Chile: Analysis of regulations, environmental impacts and bioremediation systems. Coastal and Ocean Management, 52 (5): 243-249. https://doi.org/10.1016/j.ocecoaman.2009.03.002

Chopin, T., Buschmann, A. H., Halling, C., Troell, M., Kautsky, N., Neori, A., Kraemer, G. P., Zertuche-González, J. A., Yarish, C. and Neefus, C. (2001). Integrating seaweeds into aquaculture systems: a key towards sustainability. Journal of Phycology, 37: 975-986. https://doi.org/10.1046/j.1529-8817.2001.01137.x

Chopin, T., Robinson, S. M. C., Troell, M., Neori, A., Buschmann, A. H. and Fang, J. (2008). Multitrophic Integration for Sustainable Marine Aquaculture. En: Sven Erik Jørgensen and Brian D. Fath (Editor-inChief), Ecological Engineering. Vol. 3, Encyclopedia of Ecology, 5: 463-2475. https://doi.org/10.1016/B978-008045405-4.00065-3

Chopin, T. (2013). A look at Integrated multi-trophic aquaculture. Global Aquaculture Advocate. March Issue. www.aquaculturealliance.org/advocate/look-at-integrated-multi-trophic-aquaculture

Chopin, T. \& Tacon, A. (2020). Importance of seaweeds and extractive species in global aquaculture production. Reviews in Fisheries Science \& Aquaculture, https://doi.org/10.1080/23308249.2020.1810626 
Fei, X. G. (2004). Solving the coastal eutrophication problem by large scale seaweed cultivation. Hydrobiologia 512: 145-151. https://doi.org/10.1007/978-94-007-0944-7 19

Harlin, M. M., Thorne-Miller, B. and Thursby, B. G. (1979). Ammonium uptake by Gracilaria sp. (Florideophyceae) and Ulva lactuca (Chlorophyceae) in closed system fish culture. En: Jensen, A., Stein, J.R. (Eds.), Proceedings IXth International Seaweed Symposium, Science Press, Princeton, 285-293.

Langton, R. W., Haines, K. C. and Lyon, R. E. (1977). Ammonia nitrogen produced by the bivalve mollusc Tapes japonica and its recovery by the red seaweed Hypnea musciformis in a tropical mariculture system. Helgoländer wissenschaftliche Meeresuntersuchungen, 30: 217-229. https://doi.org/10.1007/BF02207837

McDonald, M. E. (1987). Biological removal of nutrients from wastewater: an algal-fish system model. En: Reddy, K. R., Smith, W. H. (Eds.), Aquatic Plants for Waste Water Resource Recovery, Magnolia Publishing, Orlando, Florida, USA. 959-968.

Neori, A., Krom, M.D., Cohen, Y. and Gordin. H. (1989). Water quality conditions and particulate chlorophyll a of new intensive seawater fishpond in Eilat, Israel: daily and dial variations. Aquaculture, 80: 63-78.

Neori, A., Cohen, I. and Gordin, H. (1991). Ulva lactuca biofilters for marine fish-pond effluents: II. Growth rate, yield and C:N ratio. Botanica Marina 34: 483-489. https://doi.org/10.1515/botm.1991.34.6.483

Neori, A., Chopin, T., Troell, M., Buschmann, A. H., Kraemer, G. P., Halling, C., Shpigel, M. and Yarish, C. (2004). Integrated aquaculture: rationale, evolution and state of the art emphasizing seaweed biofiltration in modern aquaculture. Aquaculture 231: 361-391. https://doi.org/10.1016/j.aquaculture.2003.11.015

Petrell, R. J., Tabrizi, K. M., Harrison, P. J. and Druehl, L. D. (1993). Mathematical model of Laminaria production near a British Columbian salmon sea cage farm. Journal of Applied Phycology, 5: 1-14. https://doi.org/10.1007/BF02182416

Petrell, R. J. and Alie, S. Y. (1996). Integrated cultivation of salmonids and seaweeds in open systems. Hydrobiologia 326/327: 67-73. https://doi.org/10.1007/BF00047788

Ryther, J. H., Goldman, J. C., Gifford, J. E., Huguenin, J. E., Wing, A. S., Clarner, J. P., Williams, L. D. and Lapointe, B. E. (1975). Physical models of integrated waste recycling-marine polyculture systems. Aquaculture, 5(2): 163 - 177. https://doi.org/10.1016/0044-8486(75)90096-4

Shpigel, M. and Fridman, R. (1990). Propagation of the Manila clam Tapes semidecussatus in the effluent of marine aquaculture ponds in Eilat, Israel. Aquaculture, 90 (2): 113-122. https://doi.org/10.1016/0044$8486(90) 90335-\mathrm{K}$

Shpigel, M., Neori, A., Popper, D. M. and Gordin, H. (1993). A proposed model for "environmentally clean" landbased culture of fish, bivalves and seaweeds. Aquaculture 117 (1-2): 115-128. https://doi.org/10.1016/0044-8486(93)90128-L

Troell, M., Halling, C., Nilsson, A., Buschmann, A. H., Kautsky, N. and Kautsky, L. (1997). Integrated open sea cultivation of Gracilaria chilensis (Gracilariales, Rhodophyta) and salmons for reduced environmental impact and increased economic output. Aquaculture, 156: 45-62. https://doi.org/10.1016/S00448486(97)00080-X

Troell, M., Rönnbäck, P., Halling, C., Kautsky, N. and Buschmann, A. H. (1999). Ecological engineering in aquaculture: use of seaweeds for removing nutrients from intensive mariculture. Journal of applied Phycology, 11: 89-97. https://doi.org/10.1023/A:1008070400208

Troell, M., Halling, C., Neori, A., Buschmann, A. H., Chopin, T., Yarish C. and Kautsky, N. (2003). Integrated mariculture: asking the right questions. Aquaculture 226:(1-4): 69-80. https://doi.org/10.1016/S00448486(03)00469-1

Troell, M., Robertson-Andersson, D., Anderson,R. J., Bolton, J. J., Maneveldt, G., Halling, C. and Probyn, T. (2006). Abalone farming in South Africa: An overview with perspectives on kelp resources, abalone feed, potential for on-farm seaweed production and socio-economic importance. Aquaculture, 257 (14): 266-281 https://doi.org/10.1016/i.aquaculture.2006.02.066

Web of Knowledge [Wok]. https://apps.webofknowledge.com/WOS GeneralSearch

Xiao, X., Agusti, S., Lin, F., Li, K. Pan, Y. Yu, Y., Zheng, Y., Wu, J. and Duarte, C. M. (2017). Nutrient removal from Chinese coastal waters by large-scale seaweed aquaculture. Scientific Reports, 7: 46613. https:///doi.org/10.1038/srep46613

Yang, M. Y., Chai, Z., Wang, Q., Chen, W., He, Z. and Jiang, S. (2015). Cultivation of the seaweed Gracilaria in Chinese coastal waters and its contribution to environmental improvements. Alga Research, 9: 235244. https://doi.org/10.1016/j.algal.2015.03.017 